\title{
Application of Wavelets to Acoustic Resonance -- Elastic Targets Surrounded by Biologics
}

\author{
JoEllen Wilbur and Steven G. Kargl \\ Research and Technology Department \\ Naval Surface Warfare Center \\ Dahlgren Division/Coastal Systems Station \\ 6703 West Highway 98, Panama City, FL 32407-7001
}

\begin{abstract}
Wavelet analysis for elastic response characterization is applied to the acoustic backscattering from submerged elastic shells. Wavelet decomposition on the transfer function of a target has been applied to generalized target description in radar, which is founded on a geometrical description of the target. In this work, the application of wavelets is to study the resonance characteristics of an elastic target and more specifically the leaky Lamb waves in the acoustic scattering response from a thin shell. The paper also addresses the application of wavelets to the acoustic backscattering from submerged thin shells surrounded by dispersive scatterers, e.g. biologics. The subsonic branch of the lowest order antisymmetric leaky Lamb wave contributes strongly to the backscatter from thin spherical shells in a frequency range designated as the midfrequency enhancement. This prominent contribution in the free-field backscattering from the shell is easily isolated by wavelet decomposition. Likewise, a wavelet decomposition can extract the midfrequency enhancement in the scattered field when the shell is surrounded by a diffuse collection of non-elastic scatterers.
\end{abstract}

\section{INTRODUCTION}

Much work has been reported on the temporal and spectral description of acoustic resonance scattering from thin shells submerged in fluid[ 1 and references therein]. The acoustic backscatter caused by the disturbance on the surface of a submerged elastic shell is known to maintain relevant resonance characteristics which constitute a shell signature. Wavelet decomposition on the transfer function of a target has been applied to generalized target description in radar, which is founded on a geometrical description of the target $[2,3]$. In this paper, the application of wavelets differs in that its emphasis is to study the resonance characteristics of an elastic target and more specifically the leaky Lamb waves in the acoustic scattering response from a thin shell.

The continuous wavelet transform (CWT) forms a time-scale representation of the signal through correlation of the signal with increasingly dilated (for $a>0$ ) versions of a given function. The CWT is expressed as,

$$
\begin{aligned}
& \Psi_{x}(t, a)=x(-t) * h_{a}(t), \\
& h_{a}(t) \doteq a^{-1 / 2} h(t / a),
\end{aligned}
$$

where ${ }^{*}$, denotes convolution in $t$. By definition larger values of the scale factor, $a$, emphasize global trends in the signal time history whereas smaller values emphasize temporal details.

If the $h_{a}(t)$ are defined to satisfy the following properties

$$
\rho_{0}=\int_{0}^{\infty}\left|\mathcal{F}_{T}\left\{h_{a}(t)\right\}\right|^{2} f^{-1} d f<\infty,
$$

where $\mathscr{F}_{T}\{$.$\} denotes Fourier transformation, and$

$$
\int_{-\infty}^{\infty} h(t) d t=0,
$$

then the CWT maintains the inversion formula

$$
x(t) \stackrel{a .}{=} \rho_{0}^{-1} \int_{0}^{\infty} h_{a}(t) *, \Psi_{x}(t, a) a^{-2} d a
$$

for $x(t), h_{a}(t)$ real or analytic.

A Wigner-Ville time-frequency analysis has been studied for characterization of the surface waves on an elastic target $[4,5]$. The relation between time-scale and time-frequency analysis is well documented[6]. The WVD is defined as follows

$$
\begin{aligned}
W V D\{x(t)\} & \doteq W_{x x}(t, \omega) \\
& \doteq \int_{-\infty}^{\infty} x\left(t+\frac{\tau}{2}\right) x\left(t-\frac{\tau}{2}\right) e^{-j \omega \tau} d \tau
\end{aligned}
$$

where $x(t)$ is real. The relation between WVD analysis and that of the CWT is easily obtained as below,

$$
\begin{aligned}
|C W T|^{2} & =\left|x(-t){ }^{*}, h_{a}(t)\right|^{2} \\
& =\int_{-\infty}^{\infty} W V D\left\{x(-t) *, h_{a}(t)\right\} d \omega \\
& =\int_{-\infty}^{\infty} \int_{-\infty}^{\infty} W_{x x}(u, \omega) W_{h_{a} h_{a}}(t-u, \omega) d u d \omega
\end{aligned}
$$

In Section II wavelet analysis of the impulse response

IV-492

U.S. Government work not protected by U.S. Copyright 
of submerged spherical shells of differing shell thickness are compared in light of the application potential to elastic resonance based characterization. System properties of the continuous wavelet transform are discussed in Section III in light of their relevance to analysis of acoustic backscatter from elastic targets. The CWT is used to identify the contribution of the subsonic branch of the lowest order antisymmetric Lamb mode to the backscattering from a thin shell submerged in water where the shell is surrounded by diffuse scatterers whose target strengths emulate tuna. The problem is important to the characterization of elastic targets surrounded by biologics.

\section{ACOUSTIC RESONANCE CHARACTERIZA- TION}

The steady-state backscattered pressure in the far field of an evacuated elastic spherical shell is

$$
p(t)=\operatorname{Re}\left\{p_{0} f\left(k r_{0}\right) \frac{r_{0}}{2 R} e^{j(k R-\infty x)}\right\}
$$

where $k r_{0}=2 \pi r_{0} / \lambda ; \lambda$ is the wavelength of the incident sound; $r_{0}$ is the outer radius of the shell; $p_{0}$ is the incident pressure amplitude; $R$ is the radial distance from the shell; and $f\left(k r_{0}\right)$ is the backscattering form function of the shell given by

$$
f\left(k r_{0}\right)=\frac{2}{j k r_{0}} \sum_{n=0}^{\infty}(-1)^{n}(2 n+1) \frac{B_{n}\left(k r_{0}\right)}{D_{n}\left(k r_{0}\right)} .
$$

$B_{n}\left(k r_{0}\right)$ and $D_{n}\left(k r_{0}\right)$ are $5 \times 5$ determinants whose elements are complicated linear combinations of spherical Bessel and Hankel functions of the first kind. The interested reader is referred to ref[7] for these expressions.

The acoustic backscatter from a submerged thin shell satisfies linear acoustics. A form function calculation is then possible through superposition of $\mathrm{CW}$ solutions to (10), from which the dimensionless impulse response is generated through Fourier transformation.

Figure 1 gives the impulse response for a steel spherical shell submerged in water where the shell's thickness to outer radius ratio is $2 \%$. The initial time is indicated by $t_{0}$, where the impulse at $t_{0}$ corresponds to the specular reflection. The return immediately following the specular is partially attributed to the $s_{1}$ symmetric leaky Lamb wave and partially due to transmitted bulk waves undergoing multiple reflections between shell surfaces. The $s_{1}$ contribution is often referred to as the "quasi-thickness resonance." The echoes designated by $s_{0}$ are due to the lowest symmetric Lamb wave. The echo labeled $a_{1}$ is tentatively associated with the $a_{1}$ supersonic anti-symmetric Lamb wave. The midfrequency enhancement due to the subsonic branch of the lowest order antisymmetric leaky Lamb mode is labeled $a_{0}$. The midfrequency enhancement corresponds to a frequency region where $a_{0}$. imparts a prominent wave packet in the far field impulse response of the shell[8]. The midfrequency enhancement is characterized in the transfer function by narrowband tones atop a broad nearly Gaussian spectrum. The center frequency of the Gaussian is inversely related to the shell diameter and thickness.
The impulse response for a $5 \%$ shell of equal diameter is given in Figure 2. The transient behavior of the lowest order symmetric Lamb wave becomes more pronounced and the midfrequency enhancement region moves to lower frequencies as shell thickness increases.

Figures 3 and 4 give the respective wavelet transforms of the impulse response functions for $2 \%$ and $5 \%$ spherical shells for 5 cycle cosine modulated Gaussian wavelets of the form,

$$
\begin{aligned}
& h(t)=e^{-(2 \omega t)^{2} n} \cos \left(\omega_{0} t\right) \operatorname{rect}(t / L), \\
& a=a_{0}^{n} ; \quad n=0,1,2, \ldots
\end{aligned}
$$

where $a_{0}=1.2, \alpha=20$, and $\omega_{0}$ was chosen to correspond to $\mathrm{a} k r_{0}$ of 552 .

The ridge along all dilations in each figure corresponds to the unit impulse to which the specular reflection in acoustic backscattered data is attributed. The returns of the lowest symmetric Lamb wave for the $2 \%$ shell occur in regular time intervals in the CWT and can be seen to form a ridge along $a$ indicating the $s_{0}$ resonance to be relatively transient in nature whereas the $a_{0}$. contribution is localized in the wavelet plane. The transient behavior of the $s_{0}$ Lamb waves is more pronounced with increasing shell thickness which can be seen by comparison of Figures 3 and 4 . The elastic return associated with the midfrequency enhancement appears in the CWT for scale factors corresponding to $n=11$ through $n=13$ for the $2 \%$ shell. When properly excited over the midfrequency enhancement region the shell imparts a characteristic elastic response in the backscattered return corresponding to the subsonic branch of the lowest order antisymmetric Lamb wave. The midfrequency enhancement region can be seen from Figures 3 and 4 to scale to lower frequencies with increasing shell thickness.

\section{WA VELET ANALYSIS FOR ACOUSTIC BACKSCATTER}

To interpret the wavelet transform of a backscattered acoustic return, let $y(t)$ denote the backscattered return from a thin shell with impulse response $g(t)$. Given an acoustic signal incident on the shell to be $x(t)$ such that

$$
y(t)=x(t) *, g(t)
$$

then

$$
\Psi_{y}(-t, a)=x(t) *, g(t) *, h_{a}(-t)
$$

and the commutativity of convolution yields

$$
\psi_{y}(t, a)=x(t) *, \Psi_{g}(t, a)=\psi_{x}(t, a) *, g(t) .
$$

If $x(t)$ is stochastic, then

$$
R_{y y}(t)=g(t) * g^{*}(-t) * R_{x x}(t)
$$

for $R_{x x}(t)$ and $R_{y y}(t)$ the respective autocorrelation functions 
of the incident pulse and backscattered return. From (13) then

$$
\Psi_{R_{y}}(t, a)=g(t) * g(-t) *, \Psi_{R_{z x}}(t, a)
$$

Equation (15) indicates that the CWT of the backscattered return satisfies the system relationship used in spectral analysis. That is, transformation of the CWT in (16) yields,

$$
\Psi_{R_{y}}(\omega, a)=|G(\omega)|^{2} \Psi_{R_{z x}}(\omega, a) .
$$

Figure 5 gives the acoustic return from a submerged $2 \%$ steel shell $\left(2 r_{0}=8 \mathrm{in}\right.$.) when excited by a 2 millisecond linear chirp pulse with a cosine rolloff envelope set to sweep over the lowest order modes of the symmetric and antisymmetric Lamb waves. The shell was surrounded by diffuse scatters that have target strengths approximately equivalent to that of tuna. The midfrequency enhancement region for this shell was estimated to be centered near $110 \mathrm{kHz}$. Experimental verification was accomplished by testing the shell in the absence of any scatterers via a ping-by-ping sweep of pulsed CW transmissions with a $6 \mathrm{kHz}$ passband at incremental frequencies and indicated the midfrequency enhancement to be centered near $108 \mathrm{kHz}$.

Figure 6 gives the corresponding CWT of the LFM output for $0.1 \mathrm{~ms}, 5$ cycle cosine modulated Gaussian wavelets defined in (11) for $f_{0}=150 \mathrm{kHz}, a_{0}=1.2$, and $\alpha=$ 6. The specular contribution can be seen in the CWT to form a ridge over dilations at an angle commensurate with the $4.5 \times 10^{7}$ sweep rate of the incident LFM. This agrees with (13) for the incident LFM convolved with the ridge along dilation in the CWT of the $2 \%$ shell impulse response for the highly transient component that produces the specular reflection in acoustic backscatter. The midfrequency enhancement is relatively narrowband and arises in the CWT in Figure 6 at the dilation parameter $n=2$ which corresponds to a cosine modulated wavelet centered at $104 k H z$.

\section{CONCLUDING REMARKS}

Theoretically derived form functions were used to generate the impulse response of submerged spherical shells. The form functions were used to study the application of wavelets to characterize the elastic response of the acoustic backscattering from submerged thin spherical shells. When the wavelet transform, using the modulated Gaussian, was applied to the acoustic scattering response from a thin shell the leaky Lamb mode associated with the midfrequency enhancement elastic response could be identified even when the object was surrounded by diffuse scatterers. The approach appears promising and this work will continue with attention to the design of a wavelet basis to efficiently characterize the target transfer function over the region of the excitation signal. The objective is target discrimination directly from the backscattered data.

\section{ACKNOWLEDGEMENTS}

This work was supported under a Coastal Systems Station Independent Research Task sponsored by the ONR

\section{REFERENCES}

[1] S. G. Kargl and P.L. Marston, "Ray synthesis of the form function for backscattering from an elastic spherical shell: Leaky Lamb waves and longitudinal resonances," J. Acoust. Soc. Am., vol. 86, no. 6, 1991.

[2] I. Jouny, "Target Description Using the Wavelet Transform," IEEE Int. Conf. Acoust., Speech, Sig. Processing, vol. 4, pp.289-292, 1992.

[3] P. Flandrin et.al.,"Generalized Target Description and Wavelet Decomposition," IEEE Trans. Sig. Processing, vol. 38, no. 2, pp. 350-352, 1990.

[4] P. Flandrin et. al., "Application of Time-Frequency Analysis to the Characterization of Surface Waves on Elastic Targets," Acoustics Letters, vol. 10, no. 2, 1986.

[5] J. Wilbur and R.J. McDonald, "Acoustic data signaturing using cyclic correlations derived from the Wigner distribution," J. Acoust. Soc. Am., Suppl. 1, Nov. 1990.

[6] I. Daubechies, Ten Lectures on Wavelets, CBMS-NSF Regional Conf. Series in App. Math, 61, Capital City Press, Vermont, 1992.

[7] S. G. Kargl and P. L. Marston, "Observations and modeling of the backscattering of short tone bursts from a spherical shell: Lamb wave echoes, glory, and axial reverberations," J. Acoust. Soc. Am. 85, 1014-1028 (1989); 89, 2462 (1991).

[8] G.S. Sammelmann, D.H. Trivett, and R.H. Hackman, "The acoustic scattering by a submerged spherical shell. I: The bifurcation of the dispersion curve for the spherical antisymmetric Lamb wave," J. Acoust. Soc. Am., vol. 85, pp. 114-124, 1991 


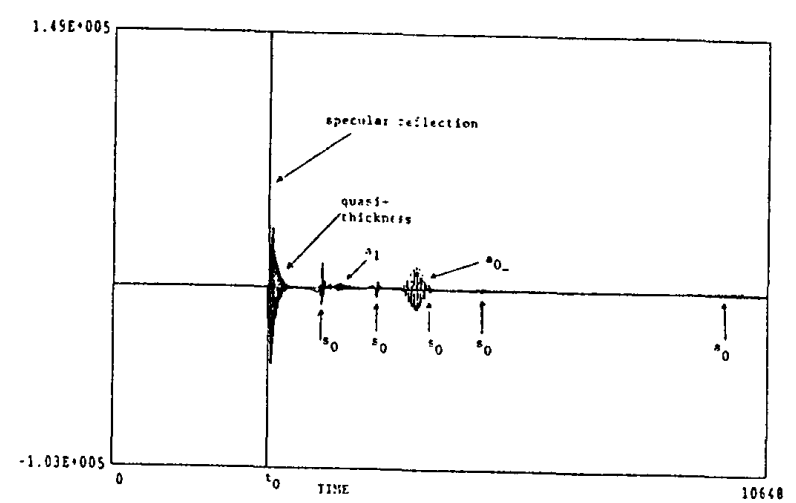

Figurc 1. Theorctically derived unit impulsc response for a stcel spherical $2 \%$ shill.

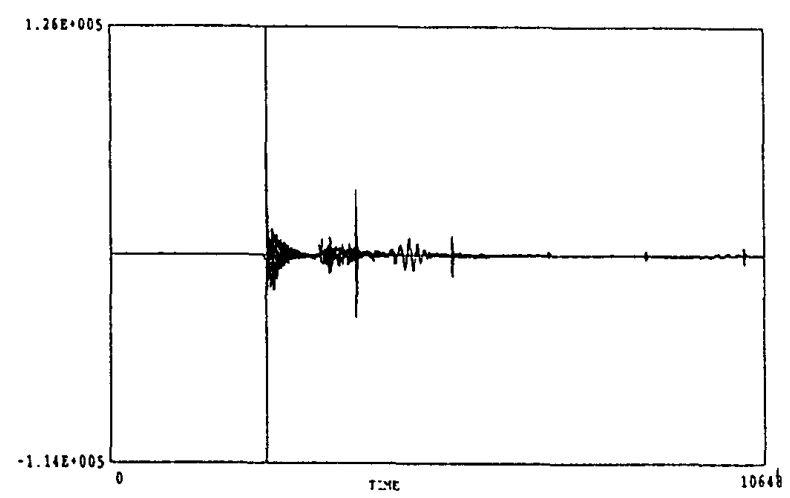

Figure 2. Theorctically derived unit impulsc response for a sicel sphcrical $5 \%$ shell.

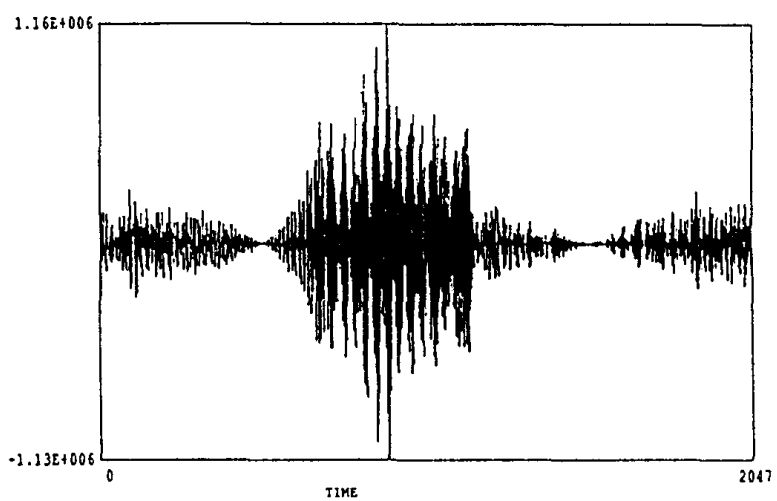

Figurc 5. Return from an 8 inch stecl shcll surrounded by diffuse scattercrs. Incident pulse $2 \mathrm{~ms}$ LFM sct to swcep from $60 \mathrm{~kJ} / \mathrm{z}$ to $150 \mathrm{k} / \mathrm{l}$. Shall thickness $2 \%$.

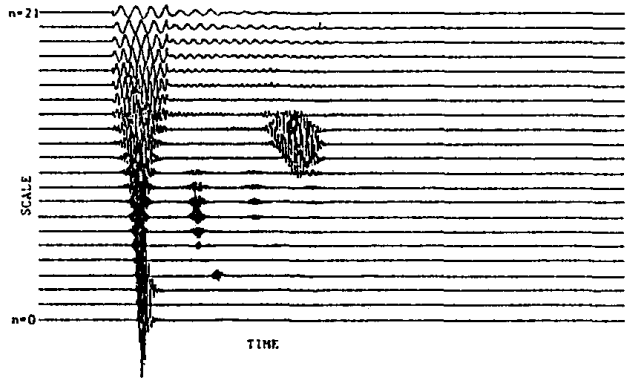

Figurc 3. Continuous wavcict transform of $2 \%$ shcll impulse response for modulated Gaussian wavelets with dilation factor $1.2^{*}, n=0,1, \ldots$ and $\omega_{b}$ corrcsponding to $a k r_{0}$ of 552 .

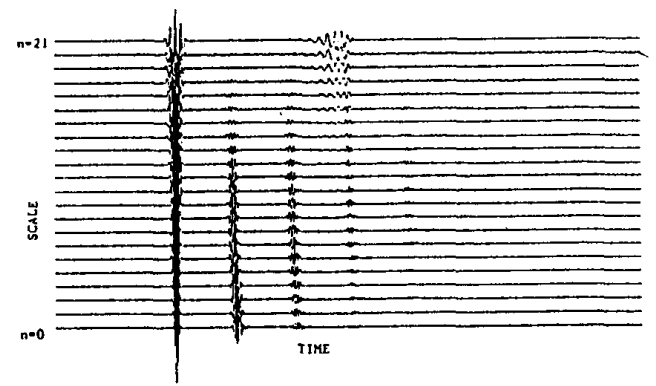

Figurc 4. Conlinuous wavelct transform of $5 \%$ shell impulse response for modulated Gaussian wavelets with dilation factor $1.2^{n}, n=0,1, \ldots$ and $\omega_{0}$ corresponding to $a r_{0}$ of 552.

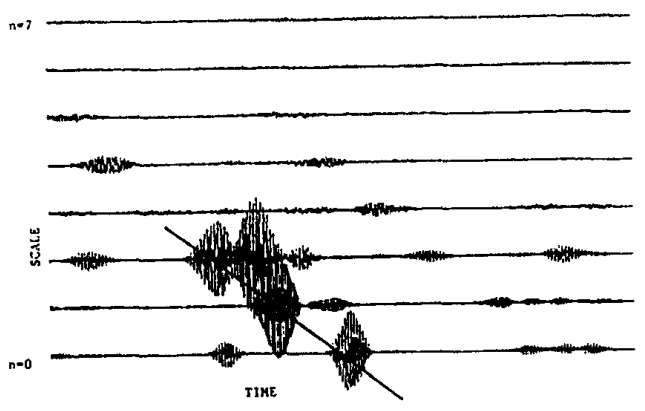

Figure 6. Continuous wavelet transform of diffuse scatterer data for $0.1 \mathrm{~ms}, 5$ cycle cosinc modulatcd Gaussian wavclets with dilation factor $1.2^{n}, n=0,1, \ldots$ and a mother wavelet carricr frequency of $150 \mathrm{kI} \mathrm{z}$. 